Comment. Math. Helv. 91 (2016), 705-719

DOI 10.4171/CMH/400
Commentarii Mathematici Helvetici

(c) Swiss Mathematical Society

\title{
Dynamics on supersingular K3 surfaces
}

\author{
Matthias Schütt* \\ Dedicated to Tetsuji Shioda on the occasion of his 75th birthday
}

\begin{abstract}
For any odd characteristic $p \equiv 2 \bmod 3$, we exhibit an explicit automorphism on the supersingular K3 surface of Artin invariant one which does not lift to any characteristic zero model. Our construction builds on elliptic fibrations to produce a closed formula for the automorphism's characteristic polynomial on second cohomology, which turns out to be an irreducible Salem polynomial of degree 22 with coefficients varying with $p$.
\end{abstract}

Mathematics Subject Classification (2010). 14J28, 14J27, 14J50, 14G17, 37C85, 37D40.

Keywords. K3 surface, automorphism, elliptic fibration, Salem number, supersingular, liftability.

\section{Introduction}

Recently, there has been a burst of activity on dynamics of K3 surfaces, in particular for supersingular ones in positive characteristic. This paper contributes to this area with rather explicit results.

The entropy of automorphisms of algebraic varieties (or Kähler manifolds over $\mathbb{C}$ ) is related to Salem polynomials. Often one aims for Salem numbers which are either small or have large degree. On K3 surfaces, the maximum degree equals the second Betti number $b_{2}=22$, but it can only be attained in two specific settings: either on non-projective complex $\mathrm{K} 3$ surfaces which contain no algebraic curves at all, as studied by McMullen [9], or on supersingular K3 surfaces in characteristic $p>0$.

A specific feature of automorphisms of maximal Salem degree on supersingular K3 surfaces was pointed out by Esnault and Oguiso [3]: such an automorphism cannot lift to any characteristic zero model of the K3 surface. Esnault and Oguiso illustrated this phenomenon explicitly with the Fermat quartic in characteristic 3, building on work of Kondō and Shimada [8]. On the implicit side, there are nonliftability results for the supersingular K3 surface $X(p)$ of Artin invariant $\sigma=1$ in characteristic $p \neq 5,7,13$ in $[2,4]$. Our aim is to exhibit non-liftable automorphisms on an infinite series of supersingular $\mathrm{K} 3$ surfaces in an explicit and systematic manner:

\footnotetext{
${ }^{*}$ Funding by ERC StG 279723 (SURFARI) is gratefully acknowledged.
} 
Theorem 1.1. For any odd prime $p \equiv 2 \bmod 3$, there exists an explicit $g \in$ $\operatorname{Aut}(X(p))$ of Salem degree 22. No power $g^{r}(r \in \mathbb{Z} \backslash\{0\})$ lifts to any characteristic zero model of $X(p)$.

The key ingredient of our approach consists in the theory of elliptic fibrations on K3 surfaces and in particular Mordell-Weil lattices, since these are at the same time accessible, versatile and allow for explicit descriptions of automorphisms. We will review the basics on elliptic fibrations and dynamics in the next two sections, before explaining the construction of the automorphisms proving Theorem 1.1. We point out that the techniques are flexible enough to lend themselves to the study of dynamics on other $\mathrm{K} 3$ surfaces, both over $\mathbb{C}$ and in positive characteristic, either of which we hope to pursue in future work.

\section{Elliptic fibrations}

Let $X$ be a K3 surface over an algebraically closed field $k=\bar{k}$ :

$$
\omega_{X} \cong \mathcal{O}_{X}, \quad h^{1}\left(X, \mathcal{O}_{X}\right)=0 .
$$

Classical examples are smooth quartics in $\mathbb{P}^{3}$ or more specifically Kummer surfaces, i.e. the resolution of the quotient of an abelian surface $A$ by inversion $l$ with respect to the group law (outside characteristic 2):

$$
\mathrm{Km}(A)=\widetilde{A / l}
$$

In what follows we will restrict for simplicity to elliptic K3 surfaces, although much of what is stated holds true in much greater generality (see [16]). An elliptic fibration on $X$ is a surjective morphism

$$
\pi: X \rightarrow \mathbb{P}^{1}
$$

such that the generic fiber is a smooth curve of genus 1 . Here we will only deal, without further distinction, with jacobian elliptic fibrations, i.e. the morphism $\pi$ admits a section. This makes the generic fiber $E$ into an elliptic curve over the function field $k(t)$ where $t$ denotes an affine parameter of $\mathbb{P}^{1}$. One derives an extensive correspondence between $X$ and $E$ as either determines the other (see [10]). Notably this materializes in a bijection between sections of $\pi$ and rational points of $E$. Either set thus forms a group which is usually referred to as Mordell-Weil group $\mathrm{MW}(X)$, or if we need to specify the fibration, $\mathrm{MW}(X, \pi)$. In order to endow $\operatorname{MW}(X)$ with a lattice structure following Shioda [20], we introduce the trivial lattice

$$
\operatorname{Triv}(X)=\langle\text { zero section, fiber components }\rangle \subset \mathrm{NS}(X) .
$$


As a consequence of Kodaira's classification of singular fibers [7] (or of Tate's algorithm for the non-complex setting [24]), the trivial lattice decomposes as an orthogonal sum of

- the hyperbolic plane $U$ generated by zero section $O$ and general fiber $F$, and

- negative-definite root lattices of Dynkin type $A_{n}, D_{m}, E_{l}$ generated by the fiber components after omitting the identity component (i.e. the component meeting $O$ ).

Note that $\operatorname{Triv}(X)$ is hyperbolic (i.e. of signature $(1, \operatorname{rk} \operatorname{Triv}(X)-1))$ and that the indices of the root lattices equal the number of fiber components minus one which leads to a straight forward rank formula for $\operatorname{Triv}(X)$. The key theorem for (jacobian) elliptic fibrations states that any divisor in $\mathrm{NS}(X)$ can be written as a sum of horizontal and vertical divisors, or more precisely, in terms of sections and fiber components:

Theorem 2.1 (Shioda [20]). There is an isomorphism of groups:

$$
\operatorname{MW}(X) \cong \mathrm{NS}(X) / \operatorname{Triv}(X) .
$$

It is precisely this isomorphism which will allow us to make both many automorphisms on K3 surfaces and their induced actions on cohomology (or NS $(X)$ ) explicit. To this end, we only have to add a lattice structure on the Mordell-Weil group, or rather its quotient by the torsion subgroup, by means of the orthogonal projection in $\operatorname{NS}(X) \otimes \mathbb{Q}$ with respect to $\operatorname{Triv}(X)$. Reversing the sign of the intersection pairing, this makes

$$
\operatorname{MWL}(X)=\operatorname{MW}(X) / \text { torsion }
$$

into a positive definite lattice (though not necessarily integral).

\section{Automorphisms and Salem numbers}

In this section, we will consider a K3 surface $X$ equipped with an automorphism $g \in \operatorname{Aut}(X)$. For instance, one can think of a linear transformation of the ambient projective space which preserves $X$. However, such an automorphism is necessarily of finite order since it leaves the hyperplane section invariant and thus acts on its orthogonal complement in $\mathrm{NS}(X)$, a negative-definite lattice, whence the claim can be seen as a consequence of the Torelli theorem (although it holds true in greater generality). Given an elliptic fibration, it is equally instructive to consider translation by a rational point $P$ on the generic fiber $E$ which extends to an automorphism of $X$, the order of which equals the order of $P \in E(k(t))$.

In what follows, we work with $\ell$-adic étale cohomology $H_{\text {êt }}^{2}\left(X, \mathbb{Q}_{\ell}(1)\right)$ after applying a Tate twist (in characteristic $p \neq \ell-$ over $\mathbb{C}$, we could equally well work 
with singular cohomology). For shortness, we will only write $H^{2}(X)$ and compute the characteristic polynomial

$$
\mu\left(g^{*} ; H^{2}(X)\right) \in \mathbb{Z}[x]
$$

(which is indeed independent of $\ell$ and integral by general theory). The relation with dynamics is fostered by the occurrence of Salem polynomials. A monic irreducible polynomial $f \in \mathbb{Z}[x]$ of degree $2 d$ is called Salem polynomial if it has $2 d-2$ roots on the unit circle plus two real positive roots $\alpha, 1 / \alpha$; as a convention, we denote the root with absolute value greater than 1 by $\alpha$.

Theorem 3.1. The characteristic polynomial $\mu\left(g^{*} ; H^{2}(X)\right)$ factors into cyclotomic polynomials and at most one Salem polynomial.

Over $\mathbb{C}$, the result is due to McMullen [9]. In positive characteristic, it relies on essential input of a result by Esnault and Srinivas [5] which implies that $g^{*}$ is finite on the orthogonal complement of the span of all the $g^{*}$-iterates of any polarization on $X$. In particular, $g^{*}$ can only have positive entropy on a subspace of $\mathrm{NS}(X)$ which then necessarily leads to a Salem polynomial by [3, Prop. 3.1] (which again draws on [9]).

We can now define the entropy of $g$ :

$$
h(g)=\left\{\begin{array}{rr}
0 & \text { if } \mu\left(g^{*} ; H^{2}(X)\right) \text { factors completely } \\
\text { into cyclotomic polynomials, } \\
\log \alpha & \text { if there is a Salem polynomial } f \mid \mu\left(g^{*} ; H^{2}(X)\right) \\
& \text { with real root } \alpha>1 .
\end{array}\right.
$$

We point out that this notion is consistent with the topological entropy from complex dynamics (compare [5]).

For our purposes, it will be crucial that $\mathrm{NS}(X) \otimes \mathbb{Q}_{\ell}$ embeds as a direct summand into $H^{2}(X)$ via the cycle class map. Denote the orthogonal complement of $\mathrm{NS}(X) \otimes \mathbb{Q}_{\ell}$ with respect to cup-product by

$$
T_{\ell}(X)=\left(\mathrm{NS}(X) \otimes \mathbb{Q}_{\ell}\right)^{\perp} \subset H^{2}(X) .
$$

This notation is consistent with the transcendental lattice $T(X) \subset H^{2}(X, \mathbb{Z})$ of a complex K3 surface $X$ as in that case $T_{\ell}(X)=T(X) \otimes \mathbb{Q}_{\ell}$. Clearly $g^{*}$ preserves $\mathrm{NS}(X)$, so the above direct sum decomposition of $H^{2}(X)$ is compatible with the $g^{*}$-action, and we obtain a factorisation

$$
\mu\left(g^{*} ; H^{2}(X)\right)=\mu\left(g^{*} ; T_{\ell}(X)\right) \mu\left(g^{*} ; \mathrm{NS}(X)\right)
$$

over $\mathbb{Z}$ (since $\operatorname{NS}(X)$ is a lattice over $\mathbb{Z}$ ). The crucial dynamical restriction for our purposes is the following observation which seems to be due to Oguiso in the complex case; for positive characteristic, the argument has been sketched just below Theorem 3.1. 
Theorem 3.2. If $X$ is algebraic, then a Salem polynomial can occur only on $\operatorname{NS}(X)$, i.e. it necessarily divides $\mu\left(g^{*} ; \mathrm{NS}(X)\right)$.

Over $\mathbb{C}$, we can be a little more explicit about the other factors:

Lemma 3.3. If $X$ is a complex algebraic K3 surface, then $\mu\left(g^{*} ; T(X)\right)$ is a power of a cyclotomic polynomial.

Proof. From Theorems 3.1 and 3.2, we know that $\mu\left(g^{*} ; T(X)\right)$ is a product of cyclotomic polynomials. But then the transcendental lattice $T(X)$ is endowed with a Hodge structure which is irreducible over $\mathbb{Q}$ (by definition, since $p_{g}=1$ ). Hence all the irreducible factors are the same.

Remark 3.4. There is an alternative proof without reference to Theorems 3.1 and 3.2: For an algebraic K3 surface, it is known that any automorphism $g$ acts by a root of unity on the regular 2-forms. By standard comparison theorems, this root occurs as an eigenvalue of $g^{*}$ on $H^{2}(X, \mathbb{Z})$, and by the very definition through the Hodge structure, on $T(X)$. Then the irreducibility gives the claim.

Note the immediate consequence of Theorem 3.2 that on an algebraic K3 surface in characteristic zero, an automorphism can have a Salem factor of its characteristic polynomials of degree at most 20 only. (This is the reason why for Salem degree 22, previous work was concentrating on non-projective K3 surfaces, see McMullen [9].)

K3 surfaces $X$ in positive characteristic $p>0$ come with the advantage that they allow for Salem polynomials of degree 22 attained on $\operatorname{NS}(X)$ if the latter has rank 22, i.e. if $X$ is supersingular. To see this at work, it seems to suffice with the supersingular K3 surface of Artin invariant $\sigma=1$, unique up to isomorphism by work of Ogus [13] and denoted by $X(p)$.

Theorem 3.5 (Blanc-Cantat, Esnault-Oguiso-Yu, Shimada). For any prime $p$, there is an automorphism $g \in \operatorname{Aut}(X(p))$ of Salem degree 22.

Corollary 3.6. The automorphism $g \in \operatorname{Aut}(X(p))$ from Theorem 3.5 does not lift to any characteristic zero model of $X(p)$.

We point out that the automorphisms in Theorem 3.5 are mostly implicit, i.e. there are existence results building on elliptic fibrations (in particular of maximal rank) and some group theory (see $[2,4])$. In contrast, until the completion of the first version of this paper it was only for $p=3$ that there was an explicit $g \in \operatorname{Aut}(X(p))$ of Salem degree 22 known [3], building on the calculation of $\operatorname{Aut}(X(3))$ by Kondō and Shimada [8]. In the meantime, Shimada used lattice-theoretic decriptions of involutions of double sextic models to equip any supersingular K3 surface (of any Artin invariant!) for the first 1000 primes with an automorphism of Salem degree 22 [19].

Our aim is to exhibit an infinite series of supersingular K3 surfaces, here $X(p)$ for odd primes $p \equiv 2 \bmod 3$, with explicit automorphisms of Salem degree 22. This will be achieved successively in the following sections. 


\section{Singular and supersingular K3 surfaces}

A complex K3 surface $X$ is called singular (in the sense of exceptional as opposed to non-smooth) if its Picard number attains the maximum

$$
\rho(X)=h^{1,1}(X)=20 .
$$

Singular K3 surfaces played an instrumental role in the proof of the Torelli theorem and of the surjectivity of the period map for K3 surfaces, see [22,23]. In particular, this led to the notion of a Shioda-Inose structure which relates $X$ to the product of two isogenous CM-elliptic curves $E \times E^{\prime}$ through rational degree 2 maps to the Kummer surface $\operatorname{Km}\left(E \times E^{\prime}\right)$ :

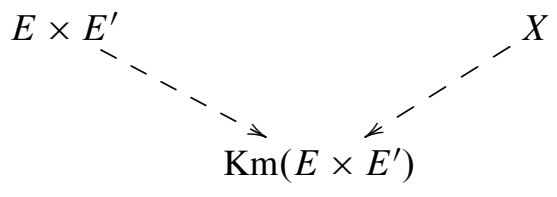

We emphasize that when working over non-closed fields, the above construction can always be carried out over a certain finite extension of the ground field (and that a singular $\mathrm{K} 3$ surface always admits a model over the ring class field $H(d)$ where $d<0$ denotes the discriminant of $\mathrm{NS}(X)$ by [14]). Therefore, classical CM theory for elliptic curves easily gives the following statement for singular K3 surfaces:

Proposition 4.1. Let $X$ be a singular $K 3$ surface, defined over some number field $F$. Let $\mathfrak{p}$ be a prime of good reduction above $p \in \mathbb{N}$. Then $X \otimes \overline{\mathbb{F}}_{\mathfrak{p}}$ is supersingular if $p$ is inert in $\mathbb{Q}(\sqrt{d})$ where $d=\operatorname{disc} \operatorname{NS}(X)$.

For all but finitely many $\mathfrak{p}$ satisfying the conditions of the proposition, the statement follows from [18]. For completeness, we include an argument which applies to all $\mathfrak{p}$ satisfying the conditions of the proposition, and in particular to those above $p=2$ where the Shioda-Inose structure breaks down.

Proof. We use modularity as exploited in [22]. Namely the zeta function of $X$ contains a quadratic factor which corresponds to the transcendental lattice. By the Shioda-Inose structure, it agrees (possibly after base extension) with some Hecke character $\psi$ of $K=\mathbb{Q}(\sqrt{d})$. By good reduction, the inertia group at $\mathfrak{p}$ acts trivially on $H_{\mathrm{et}}^{2}\left(X_{\bar{F}}, \mathbb{Q}_{\ell}\right)$, hence the transcendental lattice gives two eigenvalues $\beta, \beta^{\prime}$ of Frob ${ }_{\mathfrak{p}}^{*}$ on $H_{\mathrm{et}}^{2}\left(X_{\bar{F}_{\mathfrak{p}}}, \mathbb{Q}_{\ell}\right)$ which are compatible with $\psi$. In the ring of integers $\mathcal{O}_{K}$, we thus have

$$
\beta, \beta^{\prime} \in \mathcal{O}_{K} \quad \text { with } \quad \beta+\beta^{\prime} \in \mathbb{Z}, \quad \beta \beta^{\prime}=\mathrm{N}_{\mathbb{Q}}^{F}(\mathfrak{p})^{2} .
$$

Since $p$ is inert in $K$, it follows that both $\beta, \beta^{\prime}$ are $p$-powers times a root of unity. Hence the Tate conjecture (which holds by [1] because $X_{\bar{F}_{\mathfrak{p}}}$ carries an elliptic fibration for rank reasons) predicts that $\rho\left(X_{\bar{F}_{\mathfrak{p}}}\right)=22$. 
More precisely, it is known that any supersingular reduction $X_{\overline{\mathbb{F}}_{\mathfrak{p}}}$ will have Artin invariant $\sigma=1$, i.e. $\mathrm{NS}\left(X_{\overline{\mathbb{F}}_{\mathfrak{p}}}\right)$ has rank 22 and discriminant $-p^{2}$ by [17]. In consequence, Proposition 4.1 provides a systematic way to produce projective models for $X(p)$ for all $p$ in some given arithmetic progressions. Lattice theoretically, there is a conceptual formulation for this connection due to Shioda [21]: $X$ admits a certain elliptic fibration with section and exactly two reducible fibers, both of Kodaira type II* (unless $E$ and $E^{\prime}$ are isomorphic). This fibration which was crucial to (4.1) and is nowadays often referred to as Inose's pencil (see $[16, \S 13.5])$ comes equipped with an isometry

$$
\operatorname{MWL}(X) \cong \operatorname{Hom}\left(E, E^{\prime}\right)[2] .
$$

$\operatorname{Here} \operatorname{Hom}\left(E, E^{\prime}\right)$ is endowed with a lattice structure by means of the degree, and the intersection pairing on $\operatorname{MWL}(X)$ is that of $\operatorname{Hom}\left(E, E^{\prime}\right)$ scaled by 2. By [6], the isometry (4.2) can even be made Galois-equivariant.

\section{Isotrivial elliptic fibration}

It is rather exceptional that the whole construction from the previous section can be made explicit in terms of equations including generators of $\operatorname{NS}(X(p))$. Here we exploit one such instance: the minimal case of discriminant $d=-3$ where the singular K3 surface $X$ is most readily constructed as the minimal resolution

$$
X=\overline{(E \times E) /\left\langle\varphi \times \varphi^{2}\right\rangle}
$$

where $E$ denotes the elliptic curves with zero j-invariant and $\varphi$ an automorphism of order 3 on $E$. Projection onto either factor in $E \times E$ induces an isotrivial elliptic fibration on $X$ which has been studied from other perspectives in [15]:

$$
X: y^{2}+t^{2}(t-1)^{2} y=x^{3} .
$$

The corresponding fibration

$$
\pi: X \rightarrow \mathbb{P}_{t}^{1}
$$

has three singular fibers of Kodaira type $I V^{*}$ at $t=0,1, \infty$ (as long as the characteristic is different from 3$)$ and 3 -torsion sections $(0,0),\left(0,-t^{2}(t-1)^{2}\right)$, so that standard formulae indeed confirm rank and discriminant over $\mathbb{C}$. More precisely, fiber components and zero sections generate a sublattice

$$
U+E_{6}^{3} \subset \mathrm{NS}(X)
$$

of index 3 where the 3-divisible classes can be identified with the 3-torsion sections by Theorem 2.1. For later reference, we fix a basis of $\mathrm{NS}(X)$ starting with the general fiber and with further divisors indicated by the numbers in the following diagram of 
$(-2)$-curves on $X$ (where $7 \mathrm{a}$ and $7 \mathrm{~b}$ mean that the 7 th basis element is the sum of the two fibre components).

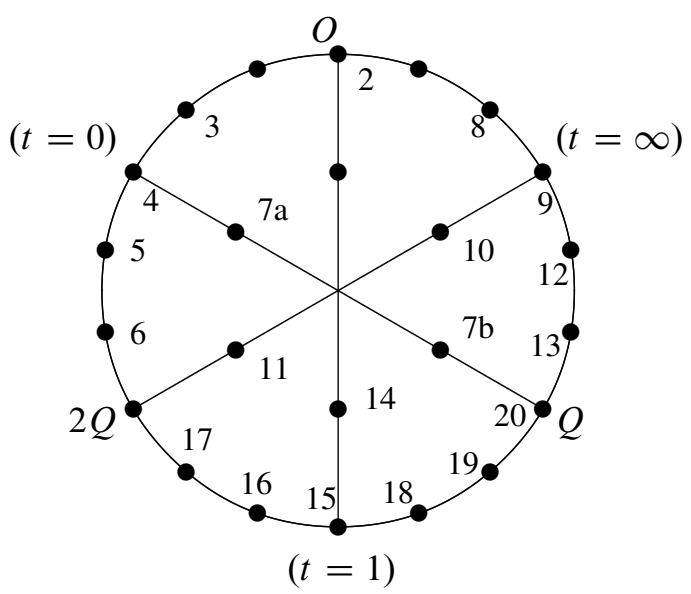

Figure 1. 24 (-2)-curves supporting singular fibers and torsion sections of $\pi$

The isotriviality allows us to pull-back $\pi$ from a rational elliptic surface $S$ by a purely inseparable base change of degree $p$ for any $p \equiv 2 \bmod 3$ (including $p=2$ ). The rational elliptic surface $S$ can be given in Weierstrass form

$$
S: y^{2}+s(s-1) y=x^{3}
$$

with singular fibers of Kodaira type $I V$ at $s=0,1, \infty$ and 3-torsion sections $(0,0),(0,-s(s-1))$. The inseparable base change leading to $X$ in characteristic $p \equiv 2 \bmod 3$ is

$$
s=t^{p} .
$$

Conceptually, this is a consequence of the specific singular fibers and their behaviour under base change; explicitly it can be derived by minimalising the resulting equation by rescaling $x$ and $y$. It follows that $X(p)$ is a Zariski surface, and we obtain $\operatorname{MWL}(X(p), \pi) \cong A_{2}^{\vee}(p)$ abstractly from the classification by Oguiso-Shioda [12] through functoriality [20, Prop. 8.12]. Alternatively, we can work out generators of $\operatorname{MW}(S)$ explicitly; for instance, the sections $P_{0}=(s, s)$ and $\omega P_{0}=(\omega s, s)$ together with the 3-torsion section $Q=(0,0)$ suffice where $\omega$ simultaneously denotes a primitive third root of unity and the corresponding automorphism of the generic fiber of (5.1) and (5.2). On the elliptic fibration given by (5.1), these induce the following sections, expressed in terms of $p=3 n+2$ :

$$
P=\left(t^{n+2} /(t-1)^{2 n}, t^{2} /(t-1)^{3 n}\right), \quad \omega P=\left(\omega t^{n+2} /(t-1)^{2 n}, t^{2} /(t-1)^{3 n}\right) .
$$


One computes the following intersection numbers between sections (symmetric in $P$ and $\omega P$ ):

$$
P . O=P . Q=P .2 Q=n, \quad P . \omega P=3 n .
$$

In addition, $P$ meets the $I V^{*}$ fibers at $t=\infty$ in the same component as $Q$ (\#13), at $t=0$ in the other non-identity component compared with $Q$ (\#6) and at $t=1$ in the identity component. In terms of the height pairing [20] one thus easily verifies that

$$
\langle P, O\rangle=\langle P, Q\rangle=\langle P, 2 Q\rangle=0, \quad\langle P, P\rangle=2 p / 3, \quad\langle P, \omega P\rangle=-p / 3,
$$

confirming $\operatorname{MWL}(X(p)) \cong A_{2}^{\vee}(p)$. Translation by $P$ on the generic fiber of (5.1) gives an automorphism

$$
\tau \in \operatorname{Aut}(X(p)) .
$$

Using Theorem 2.1, it is not hard to work out the action of $\tau^{*}$ on $\operatorname{NS}(X(p))$. For instance, $\tau^{*}$ rotates both $I V^{*}$ fibers at $t=0, \infty$ while leaving the fiber at $t=1$ invariant componentwise. We illustrate this with two more explicit examples. Complementing the above basis of $\mathrm{NS}(X)$ by $P, \omega P$ for a $\mathbb{Z}$-basis of $\mathrm{NS}(X(p))$, we can write

$$
\begin{aligned}
& P+Q=(4,2,-1,-2,-1,0,-2,-2,-4,-3,-2, \\
&-3,-2,-3,-6,-4,-2,-5,-4,-2,1,0) \\
& 2 P=(2 n+6,2,-2,-4,-2,0,-4,-2,-4,-3,-2, \\
&-3,-2,-3,-6,-4,-2,-5,-4,-3,2,0)
\end{aligned}
$$

Here is a quick guide how to find these representations: first subtract $O$ to obtain a divisor $D$ with $D . F=1$; then add and subtract fiber components until $D$ meets each fiber at exactly one component (partly predicted by the group structure on the smooth locus of the fiber); finally add a multiple of $F$ such that $D^{2}=-2$.

In exactly the same way, translation by the section $\omega P$ defines an automorphism

$$
\tau^{\omega} \in \operatorname{Aut}(X(p)) .
$$

The full matrices representing $\tau$ and $\tau^{\omega}$ in the above basis of $\operatorname{NS}(X(p))$ are available from the author's homepage; the same goes for two further automorphisms of $X(p)$ which we will develop in the next two sections, and subsequent calculation data. Together the four automorphisms will combine for the automorphism proving Theorem 1.1.

\section{Alternative elliptic fibration}

One of the keys for the implicit results in $[2,4]$ is the special feature that a $\mathrm{K} 3$ surface may admit different elliptic fibrations. For convenience, we shall only work with 
fibrations which are already visible on $X$. By work of Nishiyama [11], there are 6 such up to isomorphism, each uniquely encoded in the singular fibers.

In detail, we work with the elliptic fibration which has a fiber of Kodaira type $I_{18}$. In Figure 1, this is visible as the outer circle of $(-2)$-curves. Note that by general theory, this divisor will in fact induce the fibration

$$
\pi^{\prime}: X \rightarrow \mathbb{P}^{1}
$$

in question. Over $\mathbb{C}$, it is easy to work out $\operatorname{MW}\left(X, \pi^{\prime}\right)$ : choosing some curve as zero section $O^{\prime}$ for $\pi^{\prime}$, there is a 3-torsion section $Q^{\prime}$ and a section $R^{\prime}$ of height $3 / 2$ as indicated in Figure 2. By standard formulae for the discriminant [16, (22)], $Q^{\prime}$ and $R^{\prime}$ generate $\mathrm{MW}\left(X, \pi^{\prime}\right)$.

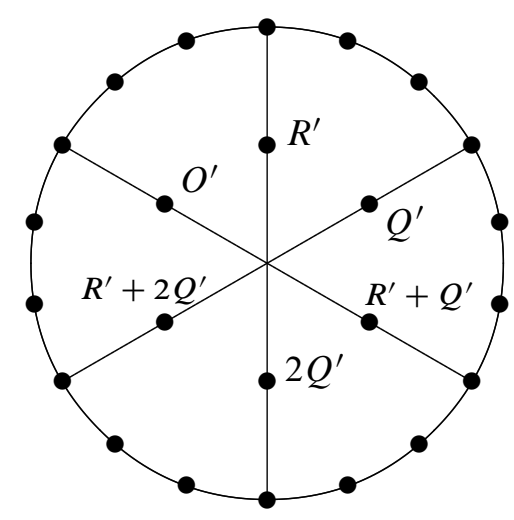

Figure 2. $I_{18}$ fiber and 6 sections

Next we consider $\pi^{\prime}$ as a fibration on $X(p)$ for an odd prime $p=3 n+2$ as above. The congruence assumption on $p$ ensures that the fibration does not degenerate (cf. Rem. 6.1), and MW $\left(X(p), \pi^{\prime}\right)$ has rank 3 (cf. Prop. 4.1). Generators complementing $Q^{\prime}, R^{\prime}$ are readily obtained from the degree $p$-multisections $P, \omega P$. By Theorem 2.1, these induce sections $P^{\prime}, \omega P^{\prime}$. For our purposes, it suffices to describe the sections as divisors in terms of the multisections and elements from the trivial lattice of $\pi^{\prime}$ as in section 5 (subtracting $(p-1) O$ and then proceeding as before).

For completeness we list the resulting intersection numbers (again symmetric in $P^{\prime}, \omega P^{\prime}$, and in perfect agreement with $\left.\operatorname{disc} \mathrm{NS}(X(p))=-p^{2}\right)$ :

$$
\begin{aligned}
P^{\prime} \cdot O^{\prime} & =\left(3 n^{2}+8 n+1\right) / 4, & P^{\prime} \cdot Q^{\prime} & =(3 n+5)(n+1) / 4 . \\
P^{\prime} \cdot R^{\prime} & =\left(3 n^{2}+2 n+3\right) / 4, & P^{\prime} \cdot \omega P^{\prime} & =3 n .
\end{aligned}
$$


As in Section 5, we can then define an automorphism

$$
\tau^{\prime} \in \operatorname{Aut}(X(p))
$$

by translation by $P^{\prime}$ on the generic fiber of $\pi^{\prime}$ and compute the induced action on NS $(X(p))$.

Remark 6.1. At $p=2$, the fibration $\pi^{\prime}$ degenerates as it attains three additional reducible fibers of Kodaira type $I_{2}$ (with fiber components given by the sections $-P$, $\left.-\omega P,-\omega^{2} P\right)$. Since $\mathrm{MW}\left(X(2), \pi^{\prime}\right) \cong \mathbb{Z} / 6 \mathbb{Z}$, the above computations cannot carry over. Indeed, on the contrary, the multisection $P$ induces the 3-torsion section $Q^{\prime}$ for $\pi^{\prime}$.

\section{Extra involution}

In order to prove Theorem 1.1, it is crucial to throw in an involution which does not respect the elliptic fibration (5.1) above. Almost to the contrary, it exploits a symmetry in Figure 1 which makes the fibration with three fibers of type $I V^{*}$ visible in two essentially different ways: the one depicted in Figure 1 and the one which features the original torsion sections $O, Q, 2 Q$ as triple components of the singular fibers:

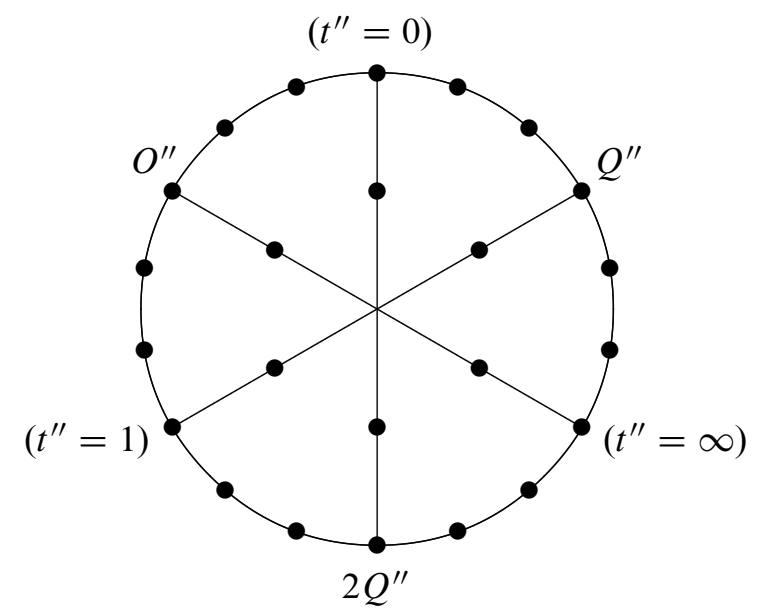

Figure 3. Isomorphic fibration with three $I V^{*}$ fibers

By the uniqueness of the fibration, there is an automorphism

$$
\imath \in \operatorname{Aut}(X)
$$

(and thus in $\operatorname{Aut}(X(p)))$ switching these two models of the fibration. Explicitly, this 
can be derived after rescaling $x, y$ in (5.1) from the resulting symmetry in $y, t$ (up to sign):

$$
X:\left(y^{2}+y\right) t(t-1)=x^{3} .
$$

For the new fibration, $P$ and $\omega P$ again induce multisections of degree $p$; modulo the trivial lattice they are equivalent to $-P,-\omega P$ in terms of the standard basis. Again, one can thus spell out the induced action of $\iota^{*}$ on $\operatorname{NS}(X(p))$.

\section{Non-liftable automorphism}

Consider the following automorphism on $X(p)$ :

$$
g=\tau^{\prime} \circ \imath \circ \tau^{\prime} \circ \imath \circ \tau^{\omega} \circ \imath \circ \tau^{\prime} \circ \imath \circ \tau^{\prime} \circ \imath \circ \tau \in \operatorname{Aut}(X(p)) .
$$

With a computer algebra system, one computes the characteristic polynomial of $g^{*}$ on $\operatorname{NS}(X(p))$ :

$$
\mu\left(g^{*}\right)=x^{11} \phi(x+1 / x),
$$

where

$$
\begin{aligned}
\phi(x)= & 6804+11016 n+2187 n^{2}+\left(-6804 n-20304+7128 n^{2}\right) x \\
& -\left(16443 n^{2}+64281 n+34254\right) x^{2}-\left(17442 n^{2}-20925 n-56020\right) x^{3} \\
& +\left(107487 n+31536 n^{2}+63852\right) x^{4}+\left(12987 n^{2}-16794 n-43992\right) x^{5} \\
& -\left(22545 n^{2}+70167 n+44646\right) x^{6}-\left(3780 n^{2}-5157 n-13414\right) x^{7} \\
& +\left(6696 n^{2}+19467 n+12900\right) x^{8}+\left(378 n^{2}-540 n-1404\right) x^{9} \\
& +\left(-1308-702 n^{2}-1929 n\right) x^{10}+x^{11}
\end{aligned}
$$

Lemma 8.1. The polynomial $\psi(x)$ is an irreducible Salem polynomial over $\mathbb{Z}$ for any $n \in \mathbb{N}$ such that $3 n+2$ is prime.

Proof. Let $n \in \mathbb{N}$ such that $p=3 n+2$ is prime. Then $\psi(x)$ comes from the automorphism $g \in \operatorname{Aut}(X(p))$ sketched above. If $\psi(x)$ were not irreducible over $\mathbb{Z}$, then it would split off a cyclotomic factor by Theorem 3.1. This, however, can be falsified simultaneously for all $n \in \mathbb{N}$ by computing the remainders after division with each cyclotomic polynomial of degree at most 22.

Proof of Theorem 1.1. We have verified for any odd prime $p \equiv 2 \bmod 3$ in Lemma 8.1 that $g \in \operatorname{Aut}(X(p))$ is an (irreducible) Salem polynomial of degree 22 . For the sake of completeness, we recall why $g$ does not lift to any characteristic zero model of $X(p)$. Assume to the contrary that there is a lift $\mathcal{X}$ of $X(p)$ over some field $K$ of characteristic zero, with some automorphism $\tilde{g} \in \operatorname{Aut}(\mathcal{X})$ lifting $g$. 
Since the isomorphism $H^{2}\left(\mathcal{X}_{\bar{K}}\right) \cong H^{2}\left(X(p)_{\overline{\mathbb{F}}_{p}}\right)$ is equivariant for the action of $\tilde{g}^{*}$ resp. $g^{*}$, this implies

$$
\mu\left(\tilde{g}^{*}\right)=\mu\left(g^{*}\right) .
$$

By Theorem 3.2, the Salem factor $\phi(x)$ can only be attained on $\operatorname{NS}\left(\mathcal{X}_{\bar{K}}\right)$, hence

$$
\rho\left(\mathcal{X}_{\bar{K}}\right)=\operatorname{deg} \phi(x)=22 .
$$

This contradicts Lefschetz' bound $\rho\left(\mathcal{X}_{\bar{K}}\right) \leq h^{1,1}\left(\mathcal{X}_{\bar{K}}\right)=20$. The same reasoning applies to any non-trivial power of $g$, since the characteristic polynomial remains irreducible of degree 22, and in particular Salem.

Remark 8.2. One can exhibit explicit automorphisms of Salem degree 22 on $X(p)$ for odd $p \equiv 2 \bmod 3$ already as a ninefold composition of our automorphisms $\tau$, $\tau^{\omega}, \tau^{\prime}, \iota$, but their characteristic polynomials turn out more complicated than $\mu\left(g^{*}\right)$. Indeed, an extensive search of compositions of the given automorphisms did not yield any irreducible characteristic polynomials of less complexity. On the other hand, we can exhibit a slightly less complicated automorphism of Salem degree 20,

$$
g^{\prime}=\tau^{\prime} \circ \imath \circ \tau^{\prime} \circ \imath \circ \tau \circ \imath \circ \tau^{\prime} \circ l \circ \tau \in \operatorname{Aut}(X(p)),
$$

which does not lift to any characteristic zero model of $X(p)$. This has characteristic polynomial $\mu\left(g^{\prime *}\right)=(x-1)(x+1) \psi(x)$ for some Salem polynomial $\psi(x)$ of degree 20. Since the latter factor has to be attained on NS of any characteristic zero lift, one can apply Lemma 3.3 to the remaining linear factors of $\mu\left(g^{\prime *}\right)$ to establish a contradiction against lifting. We emphasise, though, that this non-lifting argument does not apply to $g^{\prime 2}$.

Remark 8.3. The polynomial $\mu\left(g^{*}\right)$ is also irreducible over $\mathbb{Q}$ for $p=2$, i.e. $n=0$. However, it is not immediate to deduce that it is the characteristic polynomial of an automorphism on $X(2)$, since the fibration $\pi^{\prime}$ degenerates, see Remark 6.1.

Acknowledgements. The isotrivial fibrations first came up in discussions with Tetsuji Shioda to whom I am greatly indebted. I benefitted greatly from comments by Simon Brandhorst, Hélène Esnault, Keiji Oguiso and the anonymous referee. Thanks also to Víctor González-Alonso and Jaap Top for discussions on the subject.

\section{References}

[1] M. Artin and P. Swinnerton-Dyer, The Shafarevich-Tate conjecture for pencils of elliptic curves on K3 surfaces, Invent. Math., 20 (1973), 249-266. Zbl 0289.14003 MR 0417182

[2] J. Blanc and S. Cantat, Dynamical degrees of birational transformations of projective surfaces, J. Amer. Math. Soc., 29 (2016), 415-471. Zbl 06552401 MR 3454379 
[3] H. Esnault and K. Oguiso, Non-liftability of automorphism groups of a K3 surface in positive characteristic, Math. Ann., 363 (2015), 1187-1206. Zbl 06506162 MR 3412356

[4] H. Esnault, K. Oguiso and X. Yu, Automorphisms of elliptic K3 surfaces and Salem numbers of maximal degree, to appear in Algebraic Geometry, 2014. arXiv: 1411.0769

[5] H. Esnault and V. Srinivas, Algebraic versus topological entropy for surfaces over finite fields, Osaka J. Math., 50 (2013), 827-846. Zbl 1299.14024 MR 3129006

[6] K. Hulek and M. Schütt, Arithmetic of singular Enriques surfaces, Algebra \& Number Theory, 6 (2012), 195-230. Zbl 1248.14043 MR 2950152

[7] K. Kodaira, On compact analytic surfaces. I-III, Ann. of Math., 71 (1960), 111152; 77 (1963), 563-626; 78 (1963), 1-40. Zbl 0098.13004; Zbl 0118.15802; Zbl 0171.19601 MR 132556; MR 0184257

[8] S. Kondō and I. Shimada, The automorphism group of a supersingular K3 surface with Artin invariant 1 in characteristic 3, Int. Math. Res. Not. IMRN, 2014 (2014), no. 7, 1885-1924. Zbl 06496506 MR 3190354

[9] C. T. McMullen, Dynamics on K3 surfaces: Salem numbers and Siegel disks, J. Reine Angew. Math., 545 (2002), 201-233. Zbl 1054.37026 MR 1896103

[10] A. Néron, Modèles minimaux des variétés abéliennes sur les corps locaux et globaux, Inst. Hautes Études Sci. Publ. Math., 21 (1964), 128pp. Zbl 0132.41403 MR 0179172

[11] K.-I. Nishiyama, The Jacobian fibrations on some K3 surfaces and their Mordell-Weil groups, Japan. J. Math., 22 (1996), 293-347. Zbl 0889.14015 MR 1432379

[12] K. Oguiso and T. Shioda, The Mordell-Weil lattice of a rational elliptic surface, Comment. Math. Univ. St. Pauli, 40 (1991), 83-99. Zbl 0757.14011 MR 1104782

[13] A. Ogus, Supersingular K3 crystals, in Journées de Géométrie Algébrique de Rennes (Rennes, 1978), Vol. II, 3-86, Astérisque, 64, Soc. Math. France, Paris, 1979. Zbl 0435.14003 MR 0563467

[14] M. Schütt, Fields of definition of singular K3 surfaces, Communications in Number Theory and Physics, 1 (2007), no. 2, 307-321. Zbl 1157.14308 MR 2346573

[15] M. Schütt, Arithmetic of a singular K3 surface, Michigan Math. J., 56 (2008), 513-527. Zbl 1163.14022 MR 2488723

[16] M. Schütt and T. Shioda, Elliptic surfaces, in Algebraic geometry in East Asia (Seoul, 2008), 51-160, Advanced Studies in Pure Math., Math. Soc. Japan, Tokyo, 60, 2010. Zbl 1216.14036 MR 2732092 
[17] I. Shimada, On normal K3 surfaces, Michigan Math. J., 55 (2007), 395-416. Zbl 1136.14027 MR 2369942

[18] I. Shimada, Transcendental lattices and supersingular reduction lattices of a singular K3 surface, Trans. AMS, 361 (2009), 909-949. Zbl 1187.14048 MR 2452829

[19] I. Shimada, Automorphisms of supersingular K3 surfaces and Salem polynomials, Exp. Math., 25 (2016), no. 4, 389-398. Zbl 1342.14082 MR 3499705

[20] T. Shioda, On the Mordell-Weil lattices, Comm. Math. Univ. St. Pauli, 39 (1990), 211-240. Zbl 0725.14017 MR 1081832

[21] T. Shioda, Correspondence of elliptic curves and Mordell-Weil lattices of certain elliptic K3 surfaces, in Algebraic Cycles and Motives, Vol. 2, 319339, LMS Lect. Note Ser., 344, Cambridge Univ. Press, 2007. Zbl 1136.14028 MR 2385296

[22] T. Shioda and H. Inose, On Singular $K 3$ Surfaces, in W. L. Baily Jr., T. Shioda (eds.), Complex analysis and algebraic geometry, 119-136, Iwanami Shoten, Tokyo, 1977. Zbl 0374.14006 MR 0441982

[23] T. Shioda and N. Mitani, Singular abelian surfaces and binary quadratic forms, in Classification of algebraic varieties and compact complex manifolds, 259-287, Lect. Notes in Math., 412, Springer, Berlin, 1974. Zbl 0302.14011 MR 0382289

[24] J. Tate, Algorithm for determining the type of a singular fibre in an elliptic pencil, in Modular functions of one variable, IV, (Antwerpen, 1972), 33-52, Lect. Notes in Math., 476, 1975. Zbl 1214.14020 MR 0393039

Received January 11, 2016

M. Schütt, Institut für Algebraische Geometrie, Leibniz Universität Hannover, Welfengarten 1, 30167 Hannover, Germany

E-mail: schuett@math.uni-hannover.de 